\title{
The Discharging of Roving Objects in the Lunar Polar Regions
}

\author{
T. L. Jackson ${ }^{\text {, }}$, W. M. Farrell, ", R. M. Killen, \\ G. T. Delory ${ }^{2,4}$, J. S. Halekas ${ }^{2,4}$ \\ T. J. Stubbs $1,3,4$ \\ 1 Solar System Exploration Division, NASA Goddard Space Flight Center, Greenbelt, MD, USA \\ 2 Space Sciences Laboratory, University of California, Berkeley, CA, USA \\ 3 Goddard Earth Sciences and Technology Center, University of Maryland Baltimore \\ County, Baltimore, Maryland, USA \\ 4 NASA Lunar Science Institute, NASA Ames Research Center, Moffett Field, California, USA \\ Telana.L.Jackson@inasa.gov
}

\section{Introduction}

In 2007, the National Academy of Sciences [1] identiffed the lunar polar regions as special environments: very cold locations where resources can be trapped and accumulated. These accumulated resources not only provide a natural reservoir for luman explores, but their very presence may provide a history of lunar impact events and possibly an indication of ongoing surface reactive chemistry [2]. The recent LCROSS impacts [3] confirm that polar crater floors are rich in material including $\sim 5 \%$ wt of water.

An integral part of the special lunar polar environment is the solar wind plasma. Solar wind protons and electrons propagate outward from the Sun, and at the Moon's position have a nominal density of $5 \mathrm{el} / \mathrm{cm}^{3}$, flow speed of $400 \mathrm{~km} / \mathrm{sec}$, and temperature of $10 \mathrm{eV}(\approx 116000 \mathrm{~K})$. At the sub-solar point, the flow of this plasma is effectively vertically incident at the surface. However, at the poles and along the lunar terminator region, the flow is effectively horizontal over the surface. As recently described [4], in these regions, local topography has a significant effect on the solar wind flow.

Specifically, as the solar wind passes over topographic features like polar mountains and craters, the plasma flow is obstructed and creates a distinct plasma void in the downstream region behind the obstacle [4-7]. An ion sonic wake structure forms behind the obstacle, not unlike that which forms behind a space shuttle [8]. In the downstream region where flow is obstructed, the faster moving solar wind electrons move into the void region ahead of the more massive ions, thereby creating an ambipolar electric field pointing into the void region. This electric field then deflects ion trajectories into the void region by acting as a vertical inward force that draws ions to the surface. This solar wind 'orographic' effect is 
somewhat analogous to that occurring with terrestrial mountains. However, in the solar wind, the ambipolar E-field operating in the collisionless plasma replaces the gradient in pressure that would act in a collisional neutral gas.

Human systems (roving astronauts or robotic systems created by humans) may be required to gain access to the crater floor to collect resources such as water and other cold-trapped material. However, these human systems are also exposed to the above-described harsh thermal and electrical environments in the region. Thus, the objective of this work is to determine the nature of charging and discharging for a roving object in the cold, plasma-starved lunar polar regions. To accomplish this objective, we first define the electrical charging environment within polar craters. We then describe the subsequent charging of a moving object near and within such craters. We apply a model of an astronaut moving in periodic steps/cadence over a surface regolitl. In fact the astronaut can be considered an analog for any kind of moving human system. An astronaut stepping over the surface accumulates charge via contact electrification (tribocharging) with the lunar regolith. We present a model of this tribo-charge build-up. Given the environmental plasma in the region, we determine herein the dissipation time for the astronaut to bleed off its excess charge into the surrounding plasma.

\section{Astronauts/Objects in the Lunar Polar Electrical Environment}

As an astronaut steps along the lunar surface, charge will accumulate due to the differing contact potentials between the astronaut's suit and the surface. In essence, the astronaut becomes tribo-clarged. However, this tribo-charge can be dissipated to either the surface or the surrounding plasma. Figure 1 represents the astronaut charging as an equivalent circuit. The tribo-electric current source (represented by the switch in Fig. I) is comnected as the astronaut or object moves along the surface. There are also connections to the surface regolith and the ambient plasma.

Laboratory studies [9] indicate that the lunar rock/soil behaves as a semimconductor, with conductivity decreasing exponentially with decreasing temperature. As such, the ground conductivity drops from $10^{-6} \mathrm{~S} / \mathrm{m}$ at $1000 \mathrm{~K}$ to as low as $10^{-17} \mathrm{~S} / \mathrm{m}$ at $100 \mathrm{~K}$ [9]. Since the ground is higlily resistive in cold

polar regions (represented equivalently by resistance $R_{\mathrm{g}}$ in Fig. 1), the surrounding plasma is thus 
considered the dominant dissipation patl in these regions. In essence, the astronauts (and all human systems) are electrically-grounded to the plasma in the polar regions.

However, within polar craters, even this path becomes compromised due to the reduction of plasma currents associated witl the formation of a downstream plasma void and subsecuent ambipolar process that occurs therein. Thus, when roving in shadowed regions, such as Iunar polar craters or the night side of the moon, the plasma dissipation currents are reduced, resulting in increased dissipation times. The overall effect will lead to a clarge build-up, creating an obvious electrostatic discharge (ESD) hazard and possibly an enhancement in dust adhesion.

\section{The Electrical Environment within Polar Craters}

Now consider an object/astronaut moving near and within permanently-shadowed crater regions such as Shoemaker crater. It was recently demonstrated that lunar polar craters develop complex surface electrical environments due to the obstruction of overflowing solar wind [4]. Self-similar models of the ambipolar plasma expansion process have been presented previously [5-7], and applied to the plasma wake created belind the space shuttle in the Eartll's ionosphere [8], as well as the global-scale wake formed downstream of the Moon in the solar wind [10].

A model of the ambipolar potential that develops in Shoemaker Crater for a westward solar wind flow was previously presented in [4]. To complete our calculations, we will directly apply the polar crater plasma expansion model results in [4] to our analysis. In [4], the plasma expansion of solar wind into crater regions was based on analytical and simulation scaling. The solar wind had an electron density $n_{c o} \sim 5 \times 10^{6} / \mathrm{m}^{3}$, electron temperature $T_{e} \sim 10 \mathrm{eV}$ and flow velocity of $400 \mathrm{~km} / \mathrm{sec}$. In the primary runs, the secondary electron coefficient was 0.3. Further details of the model can be found in [4]. Figure 2 shows the total modeled potential (surface and ambipolar) relative to the solar wind and expected surface ion currents from the polar crater plasma expansion model in [4]. The top panel shows that there is the development of a region of negative potential along the leeward edge of the crater due to the lack of ion flow. There is a break in quasi-neatrality that allows electrons to flow in ahead of the ions, which creates an electron cloud region. These regions on the leeward side of the obstacle thus charge increasingly negative in order to repel electrons to compensate for the lack of ion flow. 
Exterior to the crater (topside), local currents in the model are predicted to be near $10-40 \mathrm{nA} / \mathrm{m}^{2}$ but then drop by a factor of almost 1,000 to as low as $0.04 \mathrm{nA} / \mathrm{m}^{2}$ along the leeward crater wall. In this study, we will consider the local current flow from [4] in three specific locations: (1)Topside of the crater where environmental currents have relatively large values; (2) along its leeward edge where environmental currents are the lowest; and (3) at the far edge of the crater where currents are at intermediate levels. These three locations are identified in Fig. 2.

\section{Charging Model for a Stepping Astronaut}

The lunar surface will charge in an attempt to reach an equilibritum in which the net current at the surface is zero. We assume that any object placed on the polar surface has a scale size less than the Debye length (which becomes 10's of meters approaching the lunar terminator and night side [11]), and is immersed in electron and ion flux consistent with the surface charge state. The immersed object will then charge given the particle flux contained within these currents. The local environmental currents in the sheath formed along the crater floor are given as:

$$
J_{e-c m,} \sim n_{e o} e v_{r e} \exp \left(\frac{e \phi_{T}}{k T_{o}}\right)\left(1-\delta_{e f f}\right) \sim J_{i-c m}(1)
$$

where $n_{e \omega}$ is the initial solar wind electron density, $e$ is elementary charge, $v_{t e}$ is thermal electron velocity, $k$ is the Boltzmann constant, $T_{e}$ is electron temperature, $\delta_{e f f}$ is the secondary electron coefficient of the surface, $J_{e-c m}$ and $J_{i-c m}$ are the equally-balanced local envirommental electron and ion currents, balanced by a potential $\phi_{T}$ which is a total surface/solar wind potential drop including botl the ambipolar potential drop and near-surface sheath potential drop (see [4] for details). Estimates of this envirommental current are shown in the bottom panel of Fig. 2. These currents, derived from [4], are shown here for the first time.

Now consider a dynamic situation where the astronaut is stepping. We now incorporate an impulsive tribo-charging source. In order to find the potential of the tribo-charging object relative to the local environment, a charging equation is solved: 


$$
C \frac{d \phi_{o b j}}{d t}=J_{e-m m} A\left(1-\delta_{e f f i}^{o b j}\right) \exp \left(\frac{e \phi_{a b j}}{k T_{e}}\right)-J_{i-e m,} \frac{A}{2}-C \frac{\phi_{a b j}}{\tau_{R}}+S
$$

where $\mathrm{S}$ is the tribo-charging source term, $\mathrm{C}$ is the capacitance of the system being charged, $\mathrm{A}$ is the current collecting area, $\phi_{o b j}$ is the object potential, and $\tau_{R}$ is the tribo-charge dissipation time defined by regolith permittivity and conductivity, with the $\frac{\phi_{\text {olj }}}{\tau_{R}}$ term representing the dissipation of tribo-charge into the lunar regolith [4]. We incorporate the thermal electrons (incident on all sides) and ion beam (having a specific flow and direction) by considering $\frac{A}{2}$ as the current collecting area for ions, as they are only incident on half of any exposed splzerical surface. We simulate an astronaut walking in cadence along the surface. In doing so, the astronaut periodically adds tribo-charge, which changes the object potential. This effect is modeled as a set of periodic delta functions.

$$
S=\phi_{0} C \delta(t-n \Delta t) \quad n=1,2,3, \ldots
$$

with $\phi_{0}$ being the added potential from the tribo-charge collected during each impulsive step at the boot sole/regolith interface. [The delta function produces a value only when $t=n \Delta t$. For $t \neq n \Delta t$, the function is zero.] It is shown in [12] that charge exchange from individual grain contacts with larger bodies varies as:

$$
\Delta \mathrm{q} \sim \text { Ф } \mathrm{B} 2700 \mathrm{e}
$$

where $\Phi^{\prime}$ is a normalized contact potential difference, $\Phi^{\prime}=\Delta \Phi / 2, B$ is a nomalized reduced radius $\mathrm{r}_{\mathrm{r}} / 0.5 \mu \mathrm{m}$, and $\Delta \Phi$ is the tribo-electric potential difference between the objects of differing composition (see Tribo-electric Series, Table $2[12])$. The reduced radius of the contacting grain and boot sole is $r_{r}=\left(1 / r_{g}-\right.$ 
$\left.1 / \mathrm{r}_{\mathrm{b}}\right)^{-1}$. However, the boot sole is considered very large in size compared to the grain, making the effective radius comparable to the grain radius, $\mathrm{r}_{\mathrm{r}} \sim \mathrm{r}_{\mathrm{g}}$. At the boot sole interface with the ground, we assume there is a mono-layer of grains with an average size of 100 microns $\left(A_{g} \sim 1 \times 10^{-7} \mathrm{~m}^{2}\right)$ consistent with the average regolith grain size reported by [13]. These grains are in direct contact with the $0.01 \mathrm{~m}^{2}$ boot bottom, giving $N \sim 8 \times 10^{4}$ grain/boot sole contacts in any one astronaut step. The total charge transfer per step is then $Q \sim$ $\mathrm{N} \Delta \mathrm{q}$ and the potential in equation (3) is then $\phi_{0}=\mathrm{Q} / \mathrm{C}$.

We will consider the boot sole the tribo-charging interface on the suit. The rest of the suit is exposed to the plasma. We thus solve equation (2) for two cases: the first assumes that the suit and boot sole are electrically conmected but both poor conductors (good insulators). Typically, most insulators have tribo-electric potentials similar to that of a regolith made of silica/quartz. We can thus assume that the boot sole/regolith tribo-electric potential difference is small $\left(\Phi^{\prime} \sim 0.02\right)$. Due to the poor conductivity, the accumulated charge is limited to the boot sole of radius $\mathrm{r} \sim 0.1 \mathrm{~m}$, making the sole capacitance $\mathrm{C} \sim 10 \mathrm{pF}$, and the local boot sole plasma current collecting area $\mathrm{A}=4 \pi \mathrm{r}^{2} \sim 0.12 \mathrm{~m}^{2}$. Using $\mathrm{Eq}(4)$, we find the tribo-electric charge increment at each step is $Q \sim N \Delta q \sim 10^{-10} \mathrm{C}$, adding an impulse potential of $\phi_{\mathrm{o}}=\mathrm{Q} / \mathrm{C} \sim-14 \mathrm{~V}$.

The second case assumes a conducting space suit and boot sole. Typically, conductors have large tribo-electric potential differences relative to surface silica (quartz-like material). As such we then assume a large tribo-electric potential difference between the boot sole and regolith $\left(\Phi^{\prime} \sim 2\right)$. In this case, the accumulated charge quickly spreads over the entire suit, which itself is coupled to the plasma. The astronaut body radius is assumed to be $\mathrm{r} \sim 1-\mathrm{m}$, making $\mathrm{C} \sim 100 \mathrm{pF}$, and the plasma current collecting area is thus $\mathrm{A} \sim 12 \mathrm{~m}^{2}$. Using Eq. (4) we find that the charge per step in this case is $\mathrm{Q} \sim 10^{-8} \mathrm{C}$. The impulsive potential clrange per step is thus $\phi_{0}=Q / C \sim-136 \mathrm{~V}$.

Figures $3 \mathrm{a}$ and $3 \mathrm{~b}$ show the object potential versus time, relative to the surface potential in Figure 2 , for the three test locations for an insulating space suit/boot sole; 3 a having an astronaut cadence time step of $10 \mathrm{~s}$ and $3 \mathrm{~b}$ having a time step of $2 \mathrm{~s}$. The solid lines represent the top side location, the dotted lines represent the far edge location and the dashed lines represent the crater leeward wall/edge location. Exterior to the crater, the astronaut is in direct contact with the unperturbed solar wind plasma, therefore when charge is accumulated with each step, the potential returns to equilibrium values relatively quickly. In sharp contrast, along the leeward edge, with each step, charge accumulates, but now without a clear dissipative 
path. The faster the astronaut steps, the greater the charge accumulation will be, with accumulation becoming 'perpetual'. We define perpetual charging as a situation where tlie charge accumulated per step is not efficiently dissipated in the time span between steps, thereby creating a timewaveraged charge buildmup (like that in $3 \mathrm{~b}$ ). On the far edge of the crater, with the ion deflection into the crater due to the ambipolar $\mathrm{E}_{\text {w }}$ field, charge is more easily dissipated and a 10 second cadence will allow the astronatit to return to an equilibrium charge state before taking the next step.

Figures $3 \mathrm{c}$ and $3 \mathrm{~d}$ show the object potential versus time, relative to the surface potential, for various locations, for a conducting suit; $3 \mathrm{c}$ having a time step of $10 \mathrm{~s}$ and $3 \mathrm{~d}$ having a time step of $2 \mathrm{~s}$. Again, topside of the crater, the object easily dissipates its accumulated tribo-charge to the plasma. However, within the crater, it can become highly negatively charged in a relatively short period of time, witl charging becoming perpetual with each step. At 2 second time steps, the object potential builds up to below $-2 \mathrm{kV}$ in approximately 30 seconds of movement.

\section{Recommendations}

We recommend that any space suit have a large conducting area, A, to maximize the electrical connection to the plasma and thus maintain access to the dominant charge reservoir (the electrical ground) in the polar region. However, the boot sole material has a special electrical requirement in order to remediate charge build-up: The ideal sole material should be both an electrical conductor but also have a tribo-electric potential (work function) similar to silica/quartz. Candidate materials that meet both conduction and tribo-electric requirements may come from the family of alkaline eartl metals like Ca and

$\mathrm{Mg}$. A trade study of such materials is recommended in order to determine their utility, including their reactivity. Assuming a mechanically useful material can be created, the tribo-charge accumulated per step $\left(Q=\phi_{0} C\right)$ will then be minimized but any accumulated charge on the sole will also be conducted to the large area, A, grounded to the plasma. We also recommend special care be given to venturing into regions of limited plasma flow, like the leeward edges of polar craters, since it will become difficult to have the plasma immediately dissipate/offset any tribowcharge build-up. Finally, we note that the findings herein also apply at some level to future operations of luman systems on any near-airless rocky body. While the 
material optimization may differ slightly, the same concepts of efficiently connecting to the plasma while minimizing tribo-charge build-up should still apply.

\section{Conclusions}

It is demonstrated that the ability to remediate large tribo-charge accumulations on human systems in lunar polar regions is a strong function of the envirommental plasma flux at a given location. Since the human system is primarily grounded to the plasma, as environmental currents decrease in polar craters, the dissipation of any charge build-up increases, creating a possible ESD lrazard. As evident in Fig. 3, topside regions appear to have large local envirommental currents to allow the relatively fast dissipation of tribocharge build-laps for a stepping astronaut/human system. However, clarge build-up is not fully remediated between astronaut steps inside the current-starved crater, allowing for a very large collection of charge to be accumulated on the sole of an astronaut boot.

\section{References}

[1] Paulikas, G. A. et al., "The scientific context for the exploration of the Moon," National Academies Press, Washington, DC, 2007.

[2] Crider, D. H., and Vondrak, R. R., "Space weather effects on lunar cold trap deposits," Joumal of Geophysical Research, Vol. 108,2002JIE002030, 2003.

[3] Colaprete, A. et al., "Water and more: an overview of LCROSS impact results," presented at $4 I^{\text {st }}$ Lunar and Planetary Science Conference, The Woodlands, TX, 2010.

[4] Farrell, W. M., Stubbs, T. J., Halekas, J. S., Killen, R. M., Delory, G. T., Collier, M. R., and Vondrak, R. R., "Anticipated electrical environment within permanently shadowed lunar craters," Journal of Geophysical Research, Vol. 115, E03004, doi:10.1029/2009JE003464, 2010.

[5] Crow, J. E., Auer, P. L., and Allen, J. E., "The expansion of plasma into a vacuum," Joumal of Plasma Physics, Vol. 14, No. 65, 1975.

[6] Samir, U., Wright Jr., K. H., and Stone, N. H., "The expansion of a plasma into a vacuum: Basin phenomena and processes and applications to space plasma pliysics," Reviews of Geophysics, Vol. 21, $1631,1983$.

[7] Farrell, W. M., Stubbs, T. ., Delory, G. T., Vondrak, R. R., Collier, M. R., Halekas, J. S., and Lin, R. $P$. " "Conceming the dissipation of electrically charged objects in the shadowed lunar polar regions", Geophysical Research Letters, Vol. 35, L19104, doi:10.1029/2008GL034785, 2008.

[8] Tribble, A. C., Pickett, J. S., D'Angelo, N., and Murphy, G.B., "Plasma-Density, Temperature and Turbulence in the Wake of the Sluttle Orbiter," Planetary and Space Science, Vol. 37, pp.1001-1010, 1989. 
[9] Carrier III, W. D., Olhoeft, G. R. and Mendell, W., "Physical properties of the lunar surface," Lunar Sourcebook: a user's guide to the moon, edited by G. H. Heiken, D. T. Vaniman and B. M. French, New York : Cambridge University Press, 1991, pp. 475-594.

[10] Ogilvie, K. W., Steinberg, J. T., Fitzenreiter, R. J., Owen, C. J., Lazarus, A. J.,Farrell, W. M. and Torbert, R. B., "Observations of the lunar plasma wake from the Wind spacecraft on December 27, 1994," Geophysical Research Letters, Vol. 23, No. 1255, 1996.

[11] Farrell, W. M. et al., "Complex electric fields near the lunar terminator: The near-surface wake and accelerated dust," Geophysical Research Letters, Vol. 34, L14201, doi: 10.1029/2007GL029312, 2007.

[12] Desch, S. J., and Cuzzi, J. N., "The generation of lightning in the solar nebula," Icarus, Vol. 143, No. $87,2000$.

[13] McKay D. S. et al., "The lunar regolith," Lunar Sourcebook: a user's guide to the moon, edited by G. H. Heiken, D. T. Vaniman and B. M. French, New York : Cambridge University Press, 1991, pp. 285-356.

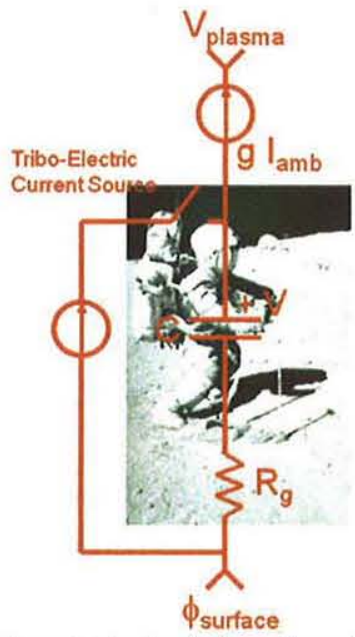

Fig. 1 Astronaut charging equivalent circuit model (Farrell et al. 2008). 


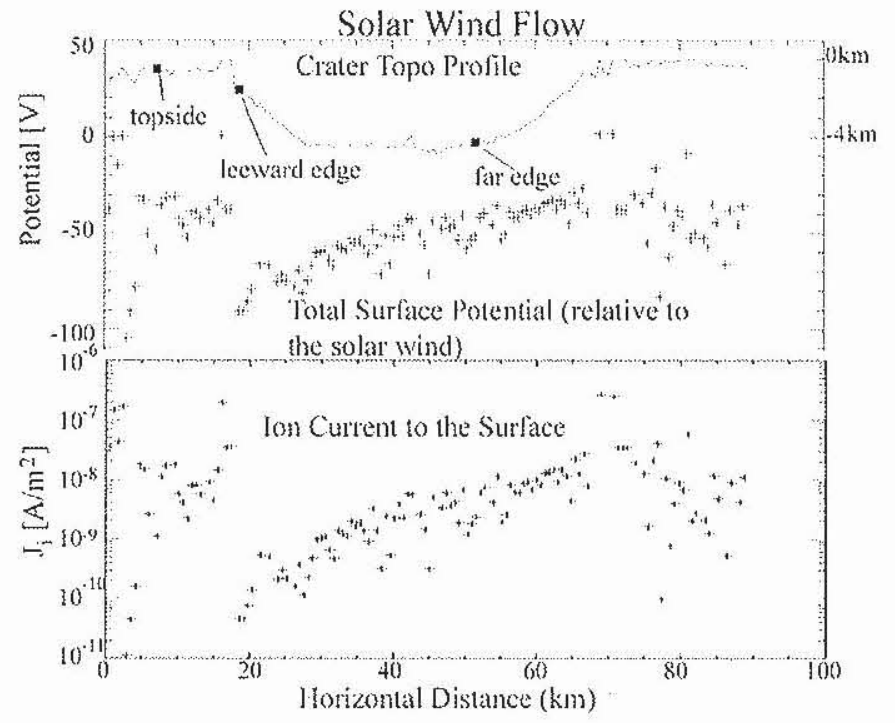

Fig. 2 'The total potential (surface and ambipolar) and ion current for westward solar wind flow over Shoemaker crater.

a)

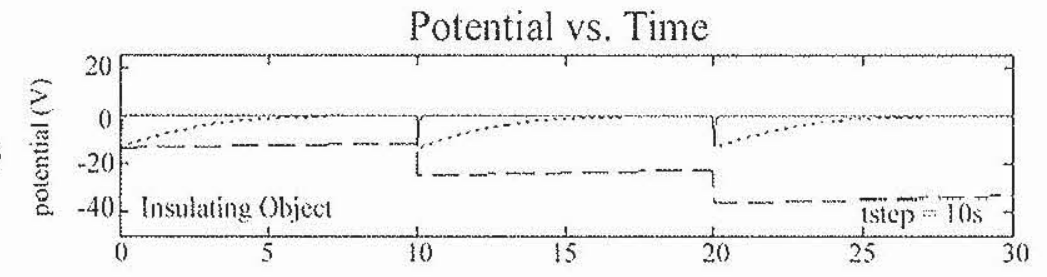

b)

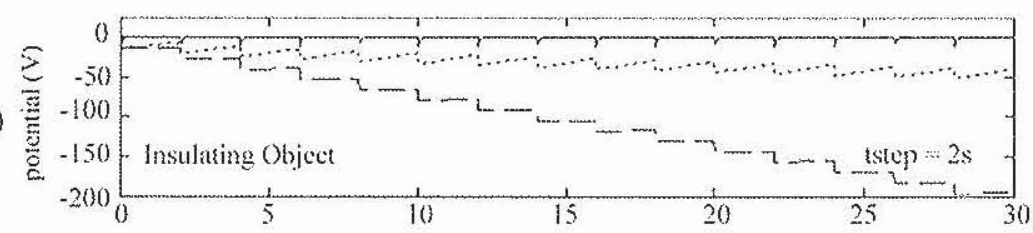

c)

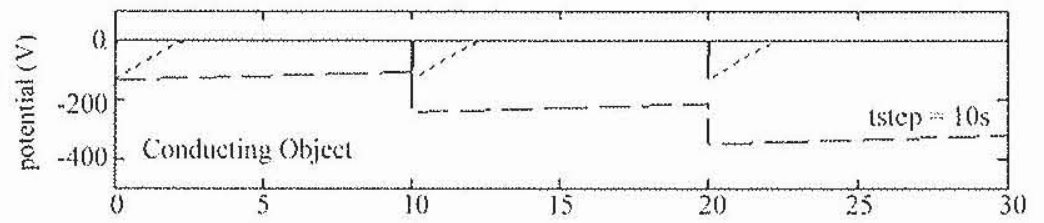

d)

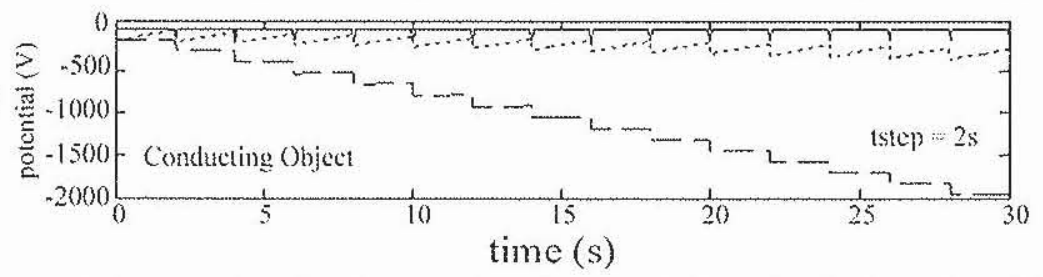

Fig. 3 Object potential versus time for three test locations for (a-b) an insulating suit and boot and (cd) a conducting suit and boot relative to the surface potential shown in Figure 2 . The solid line represents topside of the crater, the dashed line represents the leeward edge, and the dotted line represents the far edge. 